\title{
Endothelial cells microparticle-associated protein disulfide isomerase promotes platelet activation in metabolic syndrome
}

\author{
Guan-qi Fan ${ }^{1,2, *}$, Ran-ran Qin ${ }^{1,3, *}$, Yi-hui Li ${ }^{1}$, Dai-jun Song ${ }^{4}$, Tong-shuai Chen ${ }^{1}$, Wei \\ Zhang ${ }^{1}$, Ming Zhong ${ }^{1}$, Yun Zhang ${ }^{1}$, Yan-qiu Xing ${ }^{1,3}$, Zhi-hao Wang ${ }^{1,3}$ \\ ${ }^{1}$ The Key Laboratory of Cardiovascular Remodeling and Function Research, Chinese Ministry of Education and Chinese Ministry \\ of Health, The State and Shandong Province Joint Key Laboratory of Translational Cardiovascular Medicine, Department of \\ Cardiology Qilu Hospital of Shandong University, Ji'nan 250012, P.R. China \\ ${ }^{2}$ Department of Radiology Medicine, Qilu Hospital of Shandong University, Ji'nan 250012, P.R. China \\ ${ }^{3}$ Department of Geriatrics, Qilu Hospital of Shandong University, Ji'nan 250012, P.R.China \\ ${ }^{4}$ Department of Emergency, Donggang People's Hospital, Rizhao, 276800, P.R. China \\ *These authors have contributed equally to this work
}

Correspondence to: Zhi-hao Wang, email: wangzhihaosdu@126.com

Keywords: protein disulfide isomerase, platelet activation, insulin resistance, endothelial microparticles

Received: May 02, 2016

Accepted: October 17, 2016

Published: November 04, 2016

\section{ABSTRACT}

Background: Metabolic syndrome (MetS) is a common challenge in the world, and the platelet activation is enhanced in MetS patients. However, the fundamental mechanism that underlies platelet activation in MetS remains incompletely understood. Endothelial cells are damaged seriously in MetS patients, then they release more endothelial microparticles (EMPs). After all, whether the EMPs participate in platelet activation is still obscure. If they were, how did they work?

Results: We demonstrated that the levels of EMPs, PMPs (platelet derived microparticles) and microparticle-carried-PDI activity increased in MetS patients. IR endothelial cells released more EMPs, the EMP-PDI was more activated. EMPs can enhance the activation of CD62P, GPIIb/IIIa and platelet aggregation and this process can be partly inhibited by PDI inhibitor such as RL90 and rutin. Activated platelets stimulated by EMPs expressed more PDI on cytoplasm and released more PMPs.

Materials and Methods: We obtained plasma from 23 MetS patients and 8 normal healthy controls. First we built insulin resistance (IR) model of human umbilical vein endothelial cells (HUVECs), and then we separated EMPs from HUVECs culture medium and used these EMPs to stimulate platelets. Levels of microparticles, P-selectin(CD62P), Glycoprotein IIb/IIIa (GPIIb/IIIa) were detected by flow cytometry and levels of EMPs were detected by enzyme-linked immunosorbent assay (ELISA). The protein disulfide isomerase (PDI) activity was detected by insulin transhydrogenase assay. Platelet aggregation was assessed by turbidimetry.

Conclusion: EMPs can promote the activation of GPIIb/IIIa in platelets and platelet aggregation by the PDI which is carried on the surface of EMPs.

\section{INTRODUCTION}

MetS caused by IR induces hyperinsulinemia and results in a group of diseases that can cause the atherosclerosis [1]. MetS promotes platelet activation. MetS accelerates the risk of type 2 diabetes (T2DM) and cardiovascular disease considerably and it is a major cause of thrombosis, which can potentially lead to cardiocerebrovascular diseases and is related to cancer [2-4]. Thrombosis is closely related to organizational factors, platelet activation and fibrin formation, in which platelet activation is the key step of thrombosis. Platelet activation is a process of signaling cascade. The mechanism of how platelets are activated in the early process in MetS is unclear. 
PDI is a member of the thioredoxin superfamily of redoxproteins. PDI has three catalytic activities including thiol-disulfide oxidoreductase, disulfide isomerization and redox-dependent chaperone [5]. There are studies that suggest PDI may participate in platelet early activation [6]. It has been established that PDI is secreted from a variety of cell types, recently extracellular PDI has been found in platelet surface, endothelial cells and leukocytes [7]. The a and a ' domains of PDI are homologous to thioredoxin and each domain contains an independent active site. Each active site contains two cysteines in the sequence WCGHCK which mediates PDI's activities [8]. However, how PDI participates in the platelet early activation in MetS has not been reported.

There are multiple ways of platelet activation. GPIIb/IIIa (also named integrin $\alpha_{\mathrm{IIb}} \beta_{3}$ ) mediates the final way of platelet activation [9, 10]. GPIIb/IIIa receptor activation depends on the disulfide bond reduction and $\beta 3$ integrin space-conformation change [11]. Furthermore, PDI can catalysis disulfide bond reduction of GPIIb/ IIIa receptor and increase the number of sulfhydryl-free, thereby activating GPIIb/IIIa receptor into high affinity state, improving the ability to combine GPIIb/IIIa receptor with fibrinogen $[12,13]$. It is unclear whether platelets can be activated by PDI through the above way and where the PDI is from in MetS.

Membrane microparticles (MP) are shed by endothelial and blood cells upon activation or apoptosis, under the form of membrane vesicles ranging in size from 0.1 to $1 \mu \mathrm{m}$, and mostly expressing phosphatidylserine. Endothelial microparticles (EMPs) are vesicles generated by exocytic budding and display surface antigens from endothelial cells [14]. Some studies have shown that endothelial cells surface carry PDI, which can activate $\beta 1$ and $\beta 3$ integrin on platelets [15]. Platelet surface PDI (psPDI) has been studied extensively for its role in platelet activation and aggregation $[12,16]$. Platelet microparticle-associated PDI plays an important role in the platelet aggregation in T2DM and can worsen IR [17]. Therefore, we hypothesis that the endothelial cells can release PDI-containing EMPs, the PDI can activate GPIIb/ IIIa and GPIIb/IIIa is involved in platelet early activation. Meanwhile platelets released the PMPs. These steps can result in the progress of platelet signaling cascade in the condition of MetS.

\section{RESULTS}

\section{Comparison of microparticles and PDI activity in plasma}

As shown in Figure 1, the expression of CD31 $(6.03 \pm 0.27$ vs. $3.84 \pm 0.09, P<0.05)$ and CD41a $(7.32 \pm 0.37$ vs. $3.74 \pm 0.08, P<0.05)$ in plasma microparticles of MetS patients were significantly higher than the control (Figure 1A), which suggested that EMPs and PMPs increased in MetS blood circulation.

Compared with normal control, PDI enzymatic reaction curve of MetS plasma had steeper slope, rised faster, and changes of OD650nm every 10 minutes were higher $(P<0.05)$ (Figure 1B). PDI enzymatic reaction curve of MetS plasma microparticles had steeper slope, rised faster, and changes of OD650nm every 10 minutes were higher than normal control $(P<0.05)$ (Figure 1C).
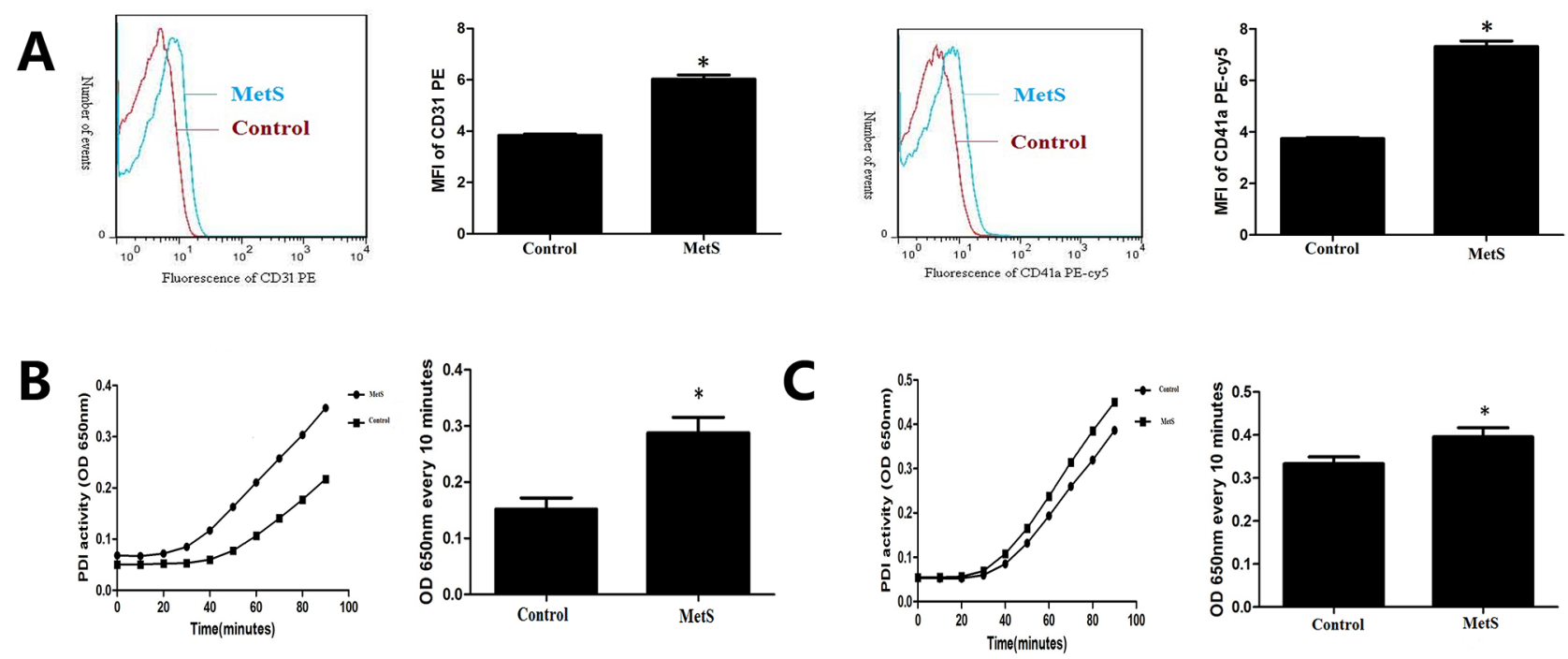

Figure 1: Comparison of microparticle and PDI activity in plasma. A. Detection of endothelial microparticles (EMPs) and platelet derived microparticles (PMPs) by flow cytometry in plasma of the control group and MetS patients. B. Detection of plasma PDI activity by insulin transhydrogenase assay of the control group and MetS patients. C. Detection of PDI activity in plasma microparticles of the control group and MetS patients. $* P<0.05$ compared with the control. Data are from at least three separate experiments. 


\section{Build IR HUVECs model}

Culture HUVECs under conditions of low glucose, low glucose combined with high insulin, high glucose and high glucose combined with high insulin. HUVECs displayed a cobblestone-like shape. There were no morphological differences between all condition HUVECs (Supplementary Figure S1). Compared with low glucose group, low glucose combined with high insulin group had similar expression of IRS1, PI3K and p-Akt $(P>0.05)$ (Figure 2A-2C). Compared with low glucose group, high glucose group had similar expression of IRS1 and PI3K $(P>0.05)$, but lower expression of p-Akt $(P<0.05)$ (Figure 2A-2C). Compared with low glucose group, high glucose combined with high insulin group had lower expression of IRS1, PI3K and p-Akt $(P<0.05)$ (Figure $2 \mathrm{~A}-2 \mathrm{C})$. Therefore, we considered high glucose combined with high insulin as condition of building IR model.

Culture HUVECs under condition of high glucose combined with high insulin for $0,2 \mathrm{~h}, 6 \mathrm{~h}, 12 \mathrm{~h}, 24 \mathrm{~h}$. Expression of IRS1 didn 't change at $2 \mathrm{~h}$ and $6 \mathrm{~h}$, decreased significantly from $12 \mathrm{~h}(P<0.05)$ and lasted stable for 24h (Figure 2D). PI3K and p-Akt, downstream of IRS1, changed in a trend of increase at first and decrease after. The inflection point happened at $6 \mathrm{~h}$ (Figures $2 \mathrm{E}-2 \mathrm{~F}$ ). These results suggested that IR happened at $12 \mathrm{~h}$ when culturing HUVECs under high glucose combined with high insulin condition and still existed at 24h. According to growth state of HUVECs, we considered high glucose combined with high insulin and $24 \mathrm{~h}$ as condition of building IR HUVECs model.

\section{IR HUVECs released EMPs carrying PDI}

Define gate of microparticles using standard fluorescent microspheres with $0.7-0.9 \mu \mathrm{m} \quad \mathrm{CD} 31$ represented endothelial cell derivation (Figure 3A). Transmission electron microscopy (TEM) images demonstrated intact EMPs of various sizes (Figure 3B). IR HUVEC released more EMPs $(P<0.05)$ by approximately 3 times than that of normal control (Figure 3C). Bicolor flow cytometry confirmed that PDI was carried by EMPs (Figure 3D). PDI enzymatic reaction curve of EMPs in supernatant of IR HUVEC medium had steeper slope, rised faster, and changes of OD650nm every 10 minutes were higher than control $(P<0.05)$ (Figure 3E). This suggested that IR EMPs had higher PDI activity.

\section{EMPs activated platelet}

EMPs stimulated platelet, MFI and percentage of platelet CD62P increased from $5 \min (P<0.05)$ and maintained a high level at $15 \mathrm{~min}$ and $30 \mathrm{~min}$ without difference with $5 \mathrm{~min}$ (Figure 4A). MFI and percentage of platelet GPIIb/IIIa increased from $15 \mathrm{~min}(P<0.05)$, and maintained a high level at $30 \mathrm{~min}$ without difference with $15 \mathrm{~min}$ (Figure 4B). This suggested that EMPs can activate platelet.
A

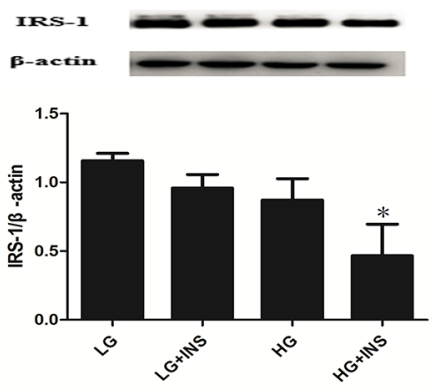

D

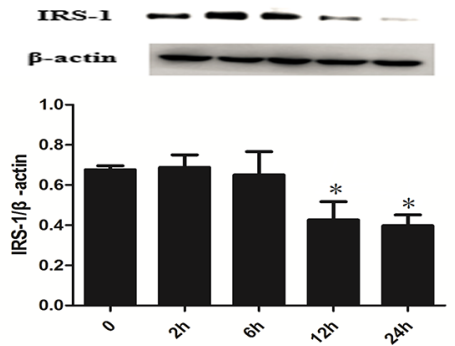

B

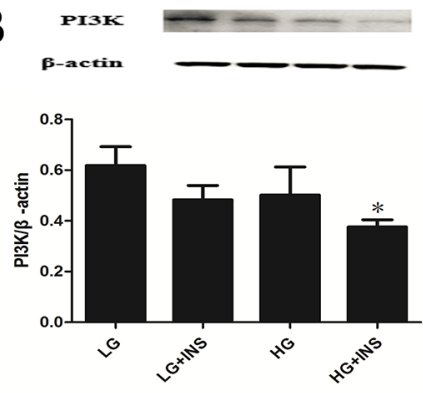

$\mathbf{E}$
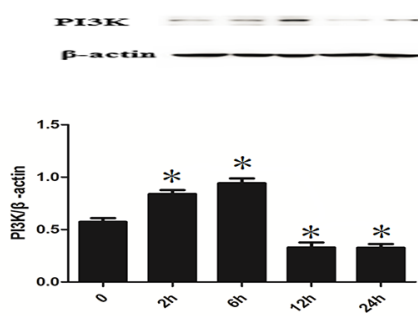
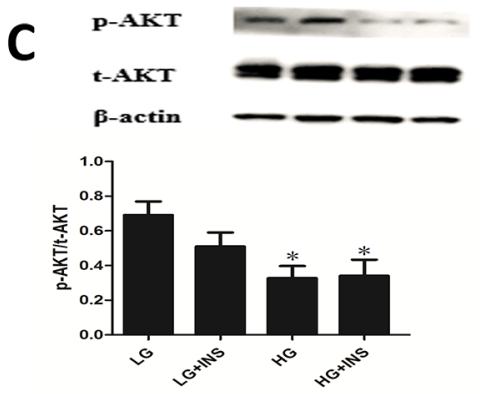

$\mathbf{F}$

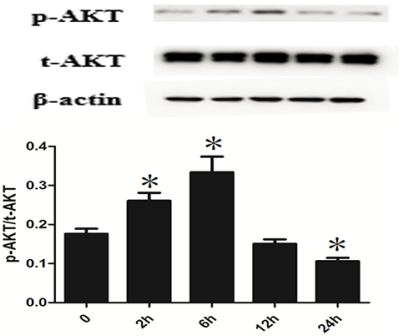

Figure 2: Build HUVECs IR model. A-C. Culture HUVECs by low glucose DMEM complete medium under conditions of low glucose(LG), low glucose combined with high insulin(LG+INS), high glucose(HG), high glucose combined with high insulin (HG+INS) for 24 hours. The expression of IRS1, PI3K and p-Akt were detected by Western blotting analysis. $* P<0.05$ compared with control. D-F. Culture HUVECs under high glucose combined with high insulin for 0, 2h, 6h, 12h, 24h,the expression of IRS1,PI3K and p-Akt were detected by Western blotting analysis. ${ }^{*} P<0.05$ compared with 0 h. Data are means \pm SD from at least three separate experiments. 


\section{Platelet stimulated by EMPs expressed PDI and released PMPs}

Platelets were stimulated with EMPs, results showed that platelet PDI expression didn't change with the stimulating time of EMPs longed $(P>0.05)$ (Figure 5A).
Stimulate platelet with EMPs and 0.5U thrombin, the results showed that platelet stimulated by EMPs and thrombin expressed more PDI than the control group $(P<$ 0.05 ) (Figure 5B). MFI and percentage of GPIIb/IIIa in PMPs increased at $15 \mathrm{~min}(P<0.05)$ and maintained a high level at $30 \mathrm{~min}$ (Figure 5C).
A
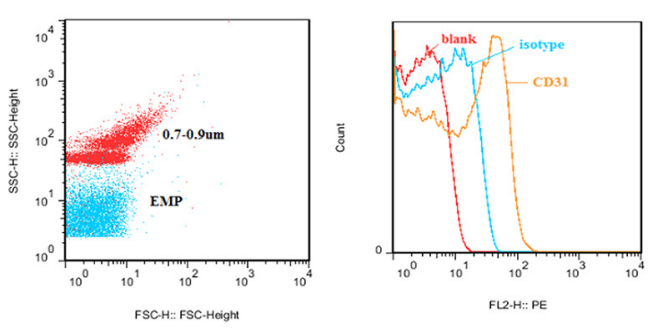

D
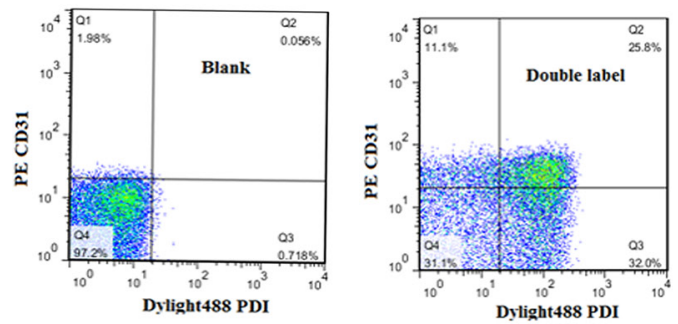

B

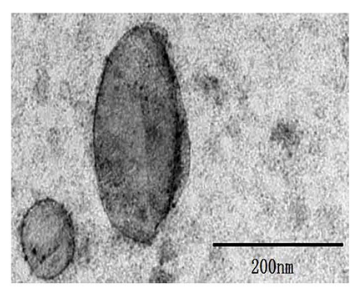

E

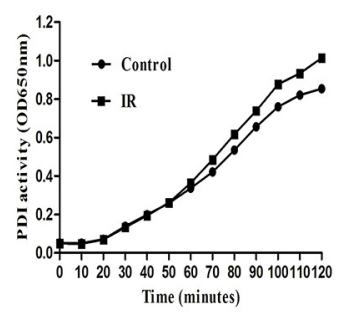

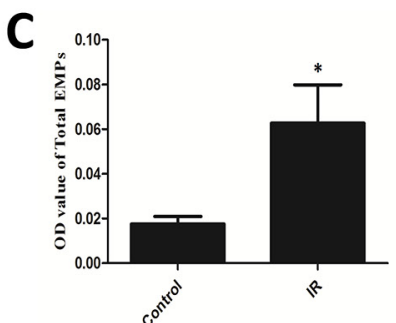

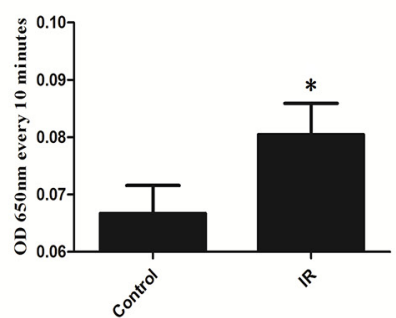

Figure 3: Detection of EMPs quantity and EMP-PDI activity. A. Define gate of microparticles using standard fluorescent microspheres with $0.7-0.9 \mu \mathrm{m}$. CD31 represented endothelial cell derivation. B. TEM images of EMPs. Scale bars 200nm. C. ELISA detected the number of EMPs in supernatant of IR and normal HUVEC medium.D. Bicolor flow cytometry confirmed EMP carrying PDI. E. ELISA combined with insulin transhydrogenase assay detected PDI activity of EMPs. ${ }^{*} P<0.05$ compared with control. Data are means $\pm \mathrm{SD}$ from at least three separate experiments.

\section{A}
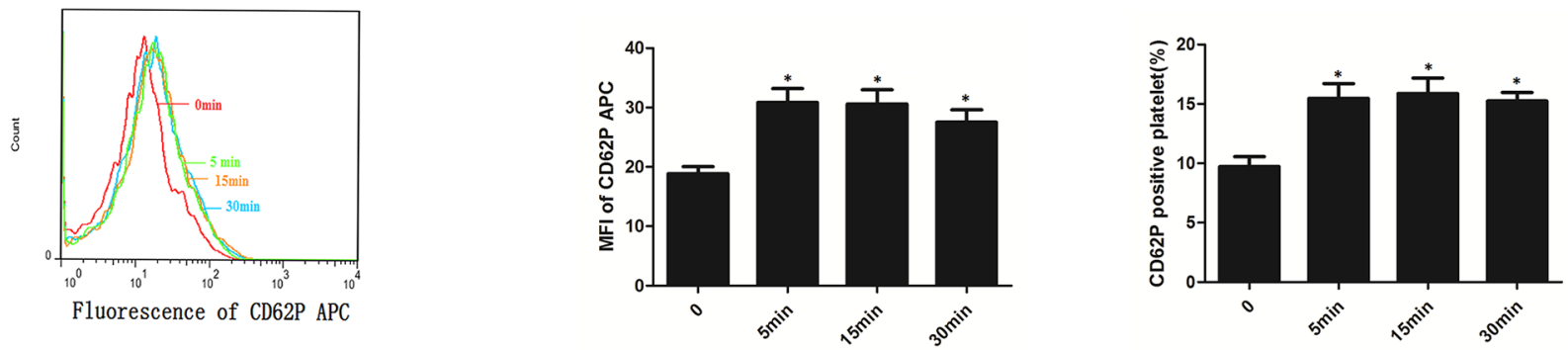

B

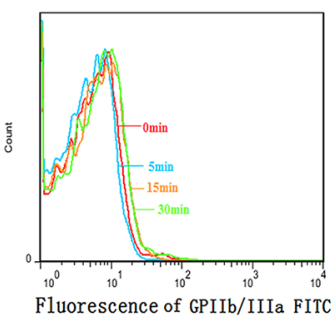

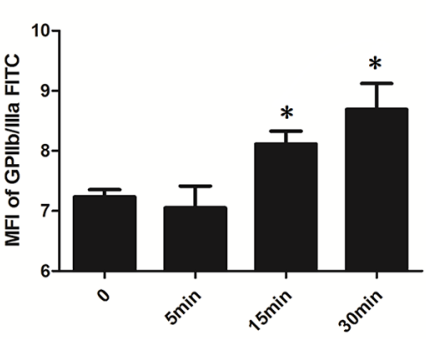

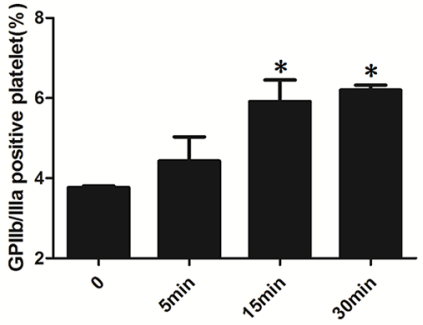

Figure 4: EMPs can activate platelet. A. Expression of CD62P of platelets which was incubated with EMPs detected by flow cytometry. B. Expression of GPIIb/IIIa of platelets which was incubated with EMPs detected by flow cytometry. Histograms represent MFI and positive. $* P<0.05$ compared with $0 \mathrm{~min}$. Data are means \pm SD from at least three separate experiments. 


\section{PDI-dependent EMPs induced platelet aggregation}

PDI enzymatic reaction curve of EMPs incubated with RL90 had gentler slope, rised slower, and changes of OD650nm every 10 minutes were lower than EMPs group $(P<0.05)$, which suggested RL90 inhibited PDI activity of EMPs (Figure 6A). Isotype (EMP incubated with IgG) had the same PDI activity with EMPs group $(P>0.05)$ (Figure 6A). Stimulate platelet with these EMPs and detect platelet aggregation. Results showed that EMPs group and isotype group had higher maximum aggregation compared with control $(P<0.05)$, EMPs incubated with RL90 had a significantly lower maximum aggregation compared with EMPs group $(P<0.05)$ (Figure 6B). These results suggested that EMPs can promote platelet aggregation and which can be inhibited with RL90.

PDI enzymatic reaction curve of EMPs incubated with rutin $(10 \mu \mathrm{M}$ and $60 \mu \mathrm{M})$ had gentler slope, rised slower, and changes of OD650nm every 10 minutes were lower than EMPs group $(P<0.05)$ (Figure 6C), which suggested different concentration of rutin inhibited PDI activity of EMPs. Stimulate platelet with EMPs incubated with $60 \mu \mathrm{M}$ rutin at $37^{\circ} \mathrm{C}$ for $30 \mathrm{~min}$ and detect platelet aggregation. Results showed that EMP group had higher maximum aggregation compared with control $(P<0.05)$, EMP were incubated with $60 \mu \mathrm{M}$ rutin had a significantly lower maximum aggregation compared with EMP group $(P<0.05)$ (Figure 6D).

\section{PDI was involved in EMP-induced platelet GPIIb/IIIa activity}

Compared with control, EMP group had more expression of PAC-1 $(P<0.05)$ and isotype group $(1 \mu \mathrm{g} / \mathrm{ml})$ had more expression of PAC-1 $(P<0.05)$. Compared with EMP group, isotype group had same expression of PAC-1 $(P>0.05)$, EMPs were incubated with RL90 group $(1 \mu \mathrm{g} /$ $\mathrm{ml})$ or rutin group $(60 \mu \mathrm{M})$ had lower expression of PAC$1(P<0.05)$, platelet pretreated with RGDS group $(10 \mu \mathrm{g} /$ $\mathrm{mL}$ ) had lower expression of PAC-1 $(P<0.05)$ (Figure 7). These results suggested that PDI was involved in EMPinduced platelet GPIIb/IIIa activity.

\section{DISCUSSION}

Our work demonstrated that the levels of EMPs and PMPs in the plasma of metabolic syndrome patients were significantly increased. At the same time, PDI activity in plasma and on microparticles were increased. More EMPs were secreted from IR HUVECs, and their corresponding PDI enzyme activity increased. EMPs activated platelets and amplified platelet activation by increasing activity of psPDI in a signaling cascade. EMPPDI could stimulate platelet activation through activating GPIIb/IIIa and this process could be partly blocked by RL90 and rutin.

Previous studies have confirmed that platelet hyper-reactivity/activation exists in MetS [18, 19]. And platelet hyper-reactivity/activation plays a central role in accelerating atherothrombosis and that is the result of the interaction among the cluster of risk factors in MetS: insulin resistance, inflammation, oxidative stress [19, 20]. A large number of studies have confirmed that endothelial dysfunction is increased and more EMPs are released in both the MetS patients and DM patients [21-24], which were consistent with our results. In addition to that, we also found that the level of PMPs increases in MetS. There are a lot of coagulation factors in the surface of particles,
A
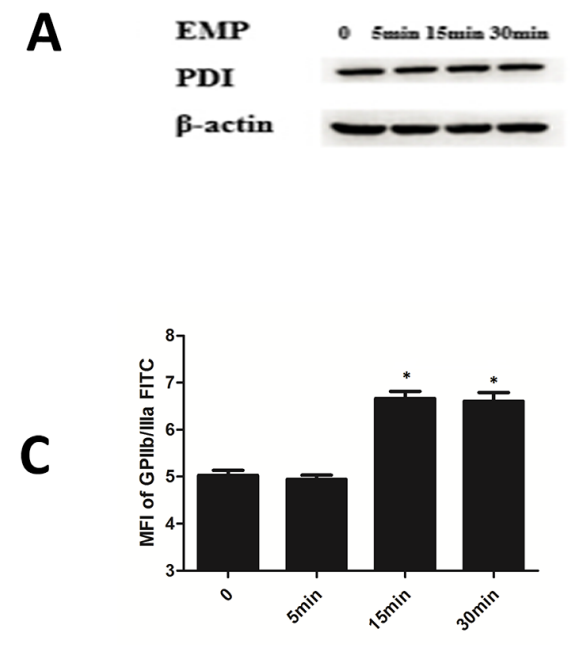
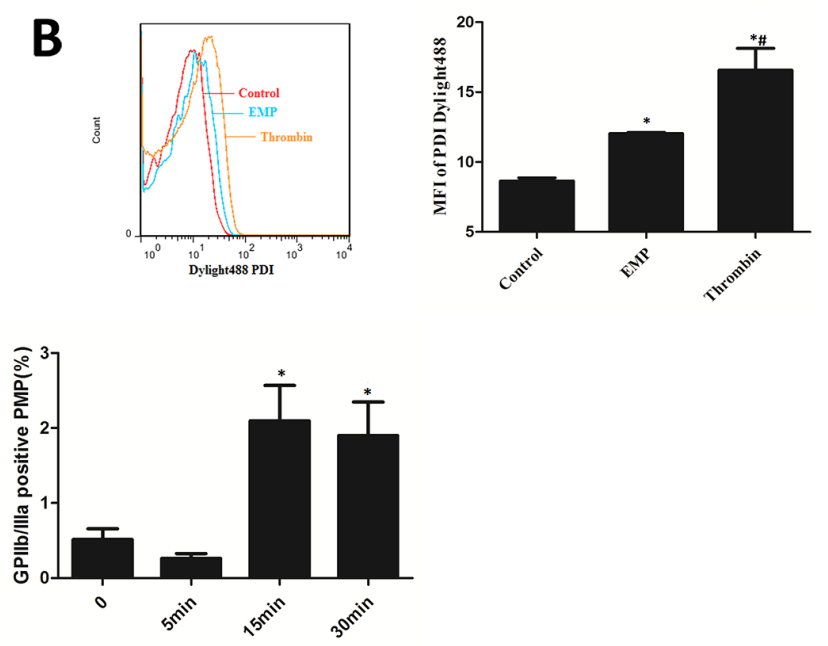

Figure 5: Platelet stimulated by EMPs expressed PDI and released PMPs. A. Platelets were stimulated with EMPs to detect the platelet PDI expression by Western blotting analysis. B. Stimulate platelet with EMPs and 0.5U thrombin to detect the platelet surface PDI by flow cytometry. Histograms represent MFI of PDI Dylight 488. ${ }^{*} P<0.05$ compared with control, ${ }^{\#} P<0.05$ compared with EMPs treatment. C. Histograms represent MFI and percentage of GPIIb/IIIa in PMPs. ${ }^{*} P<0.05$ compared with 0 min. Data are means \pm SD from at least three separate experiments. 
A
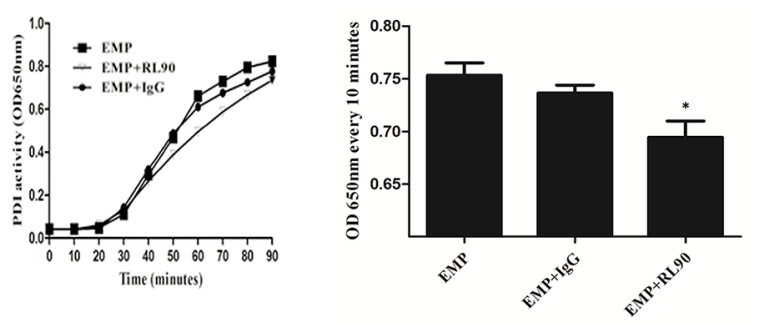

B
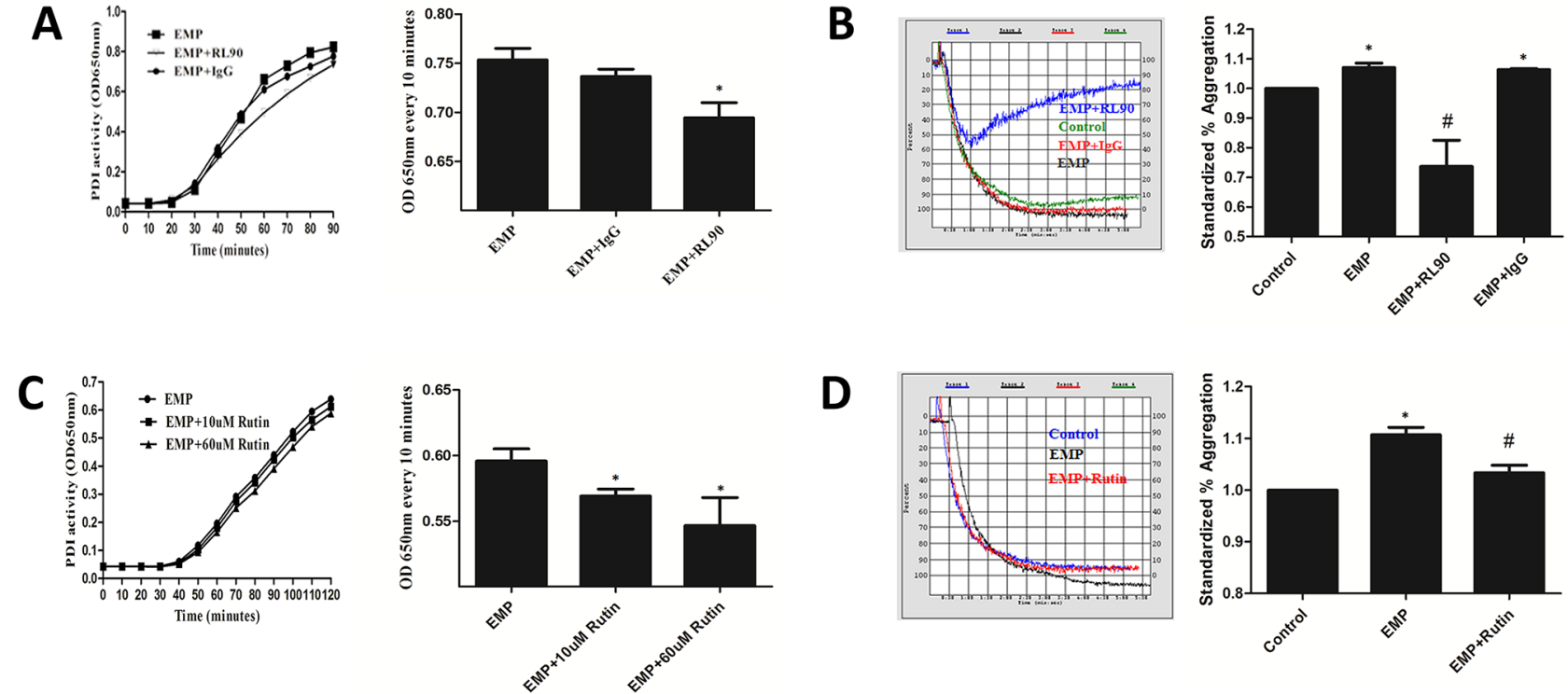
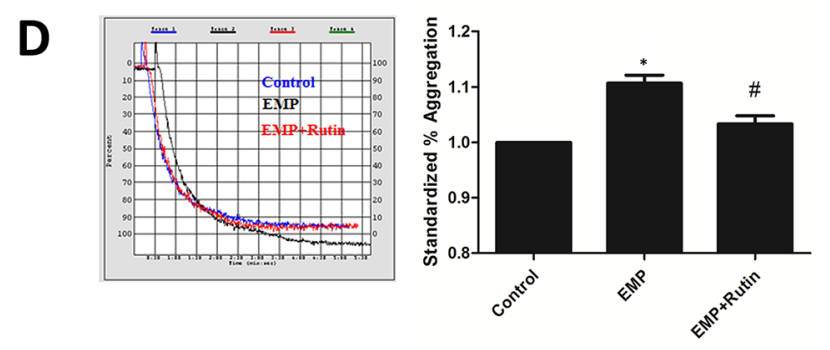

Figure 6: PDI-dependent EMPs induced platelet aggregation. Incubate EMPs with $1 \mu \mathrm{g} / \mathrm{ml} \mathrm{RL} 90, \operatorname{IgG}, 10 \mu \mathrm{M}$ and $60 \mu \mathrm{M}$ rutin at $37^{\circ} \mathrm{C}$ for $30 \mathrm{~min}$. A, C. Detect PDI activity of EMPs by insulin transhydrogenase assay, ${ }^{*} P<0.05$ compared with EMPs treatment. B, D. Detect platelet aggregation by an automatic platelet aggregation meter, ${ }^{*} P<0.05$ compared with control, ${ }^{\#} P<0.05$ compared with EMPs treatment. Data are means \pm SD from at least three separate experiments.
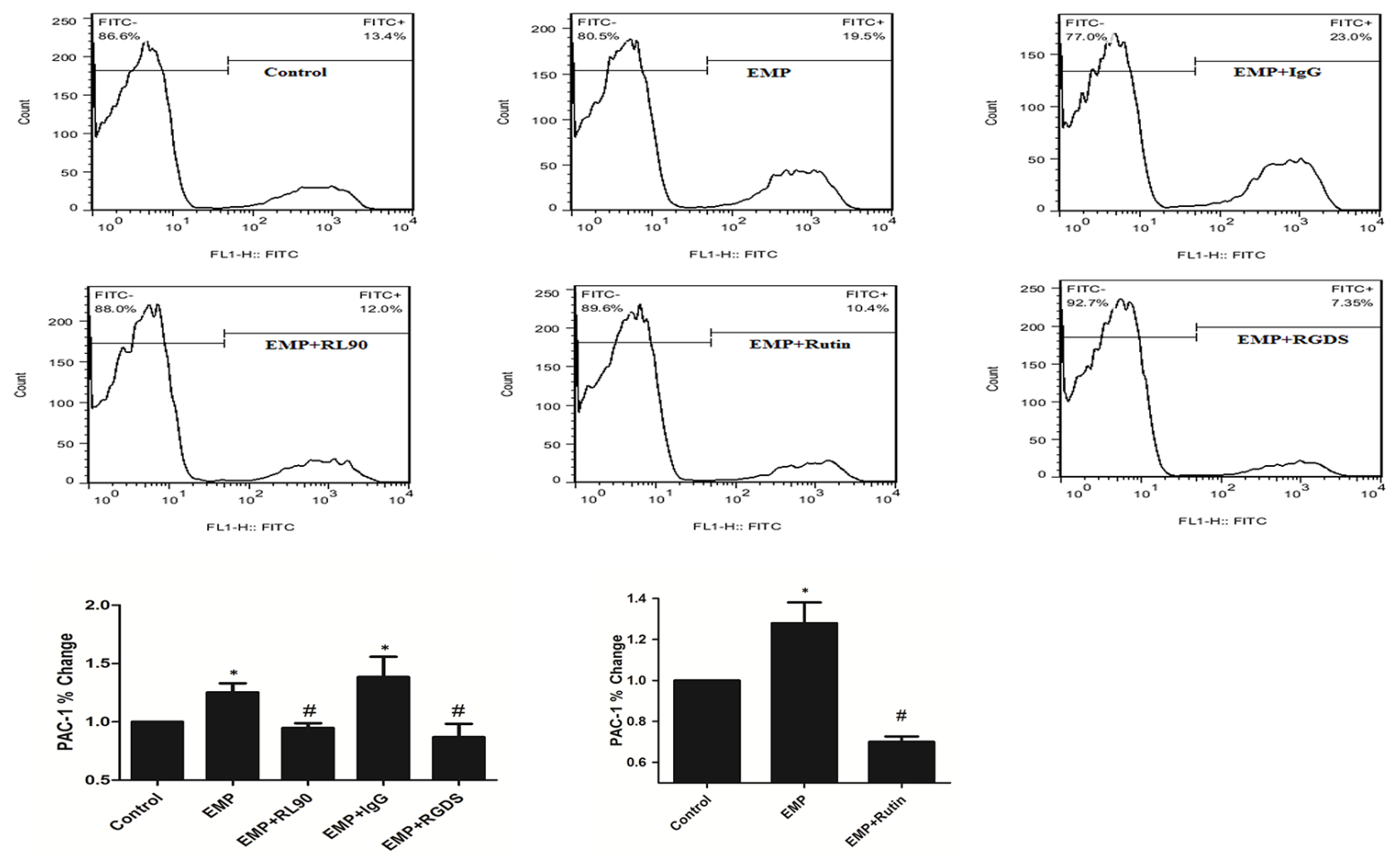

Figure 7: PDI was involved in EMP-induced platelet GPIIb/IIIa activity. Incubate EMPs with $1 \mu \mathrm{g} / \mathrm{ml} \mathrm{RL} 90, \mathrm{IgG}(1 \mu \mathrm{g} / \mathrm{mL})$, rutin $(10 \mu \mathrm{M})$, rutin $(60 \mu \mathrm{M})$ at $37^{\circ} \mathrm{C}$ for $30 \mathrm{~min}$ respectively, then stimulate platelet with the EMPs. Or incubate EMPs with RGDS (10 $\left.\mu \mathrm{g} / \mathrm{mL}\right)$ with platelet at $37^{\circ} \mathrm{C}$ for $15 \mathrm{~min}$, then incubate the platelet with EMPs. Detect platelet expression of PAC-1 (represented activated GPIIb/ IIIa) by flow cytometry. Histograms represent the PAC- $1 \%$ change. ${ }^{*} P<0.05$ compared with control, ${ }^{\#} P<0.05$ compared with EMPs treatment. Data are means $\pm \mathrm{SD}$ from at least three separate experiments. 
which can promote the platelet activation. We found that PDI activity is higher in MetS patients, which suggesting that the process of EMPs and PMPs activating platelets was correlated with PDI. Therefore EMPs may bridge the gap between endothelial dysfunction and platelet activation.

The endothelial cells will release EMPs when they are stimulated or apoptosis [23, 25, 26]. Nevertheless, it is rarely reported the field of the relationship between EMPs and platelet activation. Our study revealed that the IR HUVECs released a large number of PDI-containing EMPs, and EMP-PDI activity was significantly enhanced. When platelets were incubated with these EMPs, we found that the expression level of CD62P, PAC-1 (platelet activation markers) in these platelets were significantly increased, which suggesting that IR-EMPs can significantly activate platelets. Arun Raturi et al [17] have shown that platelet microparticle-associated PDI promoted platelet aggregation, and transfered the information of IR in T2DM. Thus, EMPs may also activate platelet through PDI and transfer the information of IR.

Integrin $\beta 3$ mediates platelet adhesion and aggregation, and the activation of integrin $\beta 3$ is the final common pathway of platelet activation. The isomerism of integrin $\beta 3$ is the key step of platelet GPIIb/IIIa activation. On the surface of platelets, GPIIb/IIIa is expressed as $\alpha_{\mathrm{IIb}} \beta_{3}[27]$, and on the surface of endothelial cells it is $\alpha_{\mathrm{V}} \beta_{3}$ not $\alpha_{\mathrm{IIb}} \beta_{3}$ [28]. PDI plays an important role in the process of platelet GPIIb/IIIa activation [29-31]. A study about the $\beta 3$-null $\left(\beta 3^{-/}\right)$mice has shown that PDI capture during thrombus formation in vivo depends on the presence of $\beta 3$ integrin [30]. This is also supported by our observation that platelets GPIIb/ IIIa activation were significantly reduced when platelets were incubated with the PDI inhibitors RL90. Therefore, we expect that PDI plays a crucial role in the activation of platelet GPIIb/IIIa and platelet aggregation. Jasuja and Furie [31] also have proved that it is the endothelium-derived but not platelet-derived PDI that is required for thrombus formation in vivo. This is consistent with our results.

Several studies have confirmed that psPDI is important for platelet release and platelet aggregation. Jasuja and Furie [31] have shown that platelet PDI contributes to the total amount of thrombus-associated PDI. And Kim Hahm et al [32] have confirmed that platelet PDI is important for thrombus formation. Our work also confirmed that when platelets were activated by EMPs, the expression of psPDI increased and platelets released more PMPs to achieve signal cascade.

\section{CONCLUSION}

GPIIb/IIIa receptor on the surface of platelets can be activated by EMP-PDI which is released by IR HUVECs, and PDI can be blocked by RL90. And platelet can express more psPDI and release PMPs to achieve signal cascade in MetS.

\section{MATERIALS AND METHODS}

\section{Materials}

The antibodies rabbit anti-RS1, anti-Akt, antiEro1 $\alpha$ and mouse anti-PI3K and anti-p-Akt were purchased from Cell Signaling Technology (Danvers, MA, USA). The antibodies mouse anti-Integrin $\beta 3$, Anti-PDI antibody (RL90), IgG isotype control and DyLight ${ }^{\circledR} 488-P D I$ were from Abcam (Boston, MA, USA). The antibody mouse anti- $\beta$-actin was purchased from Zhongshanjinqiao(Beijing, China), fluorescein isothiocyanate (FITC)-conjugated anti-PAC-1, phycoerythrin (PE) -conjugated anti-CD31, PE-conjugated isotype control, FITC-conjugated isotype control, PECy5-conjugated anti-CD41a, and allophycocyanin (APC) -conjugated anti-CD62P were purchased from BD Biosciences Pharmingen (San Diego, CA, USA). Prostaglandin $\mathrm{E}_{1}\left(\mathrm{PG}-\mathrm{E}_{1}\right)$, thrombin and RGDS (PDI inhibitor) were purchased from Sigma (Aldrich, St.Louis, MO, USA). Phosphate Buffered Saline (PBS), Bovine Serum Albumin (BSA), Dithiothreitol (DTT), and bovine insulin were purchased from Solarbio company (Beijing, China), rutin (PDI inhibitor) was purchased from Tokyo Chemical Industry (Tokyo, Japan), DMEM was purchased from Gibco Company (Carlsbad, CA, USA). Fetal Bovine Serum (FBS) was purchased from Hyclone (Logan, UT, USA). The monoclonal antibody AD-1 for microparticles was offered by Jingti Deng professor in Department of Biochemistry and Molecular Biology of University of Calgary in Canada. And analyzed on FACSCalibur and FlowJo software (BD Biosciences, San Jose, CA), microplate reader (Thermo Scientific, Waltham, MA, USA), dual channel aggregometer (Chrono-log Corp., Havertown, PA, USA), and Z2 particle counter (Beckman Coulter, Fullerton, CA, USA), transmission electron microscope (TEM) JEOL-1200EX(JEOL, Japan).

\section{Selection of clinical cases and sample collection}

Twenty-three patients with MetS and eight normal healthy controls were enrolled after appropriate inform consent was obtained. Select clinical cases according to a joint interim statement of MetS diagnosis by IDF/AHA/ free of any medication for 2 wks. Written consent was obtained from all donors, and protocols were approved by the institutional ethics committee. The subjects were fasting overnight for $8 \sim 10$ hours.

\section{Platelet preparation}

Platelet preparation is described previously [33]. Briefly, draw blood from median cubital vein of all donors to anticoagulation tubes with $0.109 \mathrm{~mol} / \mathrm{L}$ sodium citrate. Platelet-rich plasma (PRP) was prepared by centrifugation at $120 \mathrm{~g}$ for $10 \mathrm{~min}$ at room temperature. Platelets were isolated from PRP after centrifugation at $800 \mathrm{~g}$ for $10 \mathrm{~min}$ 
in the presence of acid citrate dextrose. Then platelets were washed and resuspended in modified Tyrode's buffer (137 $\mathrm{mmol} / \mathrm{L} \mathrm{NaCl}, 2.7 \mathrm{mmol} / \mathrm{L} \mathrm{KCl}, 12 \mathrm{mmol} / \mathrm{L} \mathrm{NaHCO}_{3}, 0.4$ $\mathrm{mmol} / \mathrm{L} \mathrm{NaH}_{2} \mathrm{PO}_{4}, 5 \mathrm{mmol} / \mathrm{LHEPES}, 0.1 \%$ glucose and $0.35 \%$ bovine serum albumin, $\mathrm{pH} 7.2$ ) in the presence of $100 \mathrm{nmol} / \mathrm{L}$ PG-E 1 . Platelet concentration was adjusted to $1 \times 10^{6} / \mathrm{mL}$ by use of a $\mathrm{Z} 2$ particle counter, then used at once in experiments.

\section{Human umbilical vein endothelial cell culture and build IR model}

HUVECs were presented by Gynecologic Oncology Laboratory of Qilu Hospital. Culture HUVECs by low glucose DMEM complete medium (containing 10\% FBS, $400 \mu \mathrm{l} / \mathrm{ml}$ growth factor and antibiotics). Select conditions by culture HUVECs under conditions of low glucose, low glucose combined with high insulin, high glucose, high glucose combined with high insulin. Select timing by culture HUVECs under high glucose combined with high insulin for $0,2 \mathrm{~h}, 6 \mathrm{~h}, 12 \mathrm{~h}, 24 \mathrm{~h}$.

\section{Identification of EMPs}

\section{EMPs Isolation}

EMPs were isolated from the supernatant of HUVECs after centrifugation at $15000 \mathrm{~g}$ for 1 hour at the $4^{\circ} \mathrm{C}$, in total the EMPs were resuspended in the PBS and stored at $-80^{\circ} \mathrm{C}$.

\section{Assessment of EMPs by flow cytometry and scanning electron microscopy}

The HUVEC suspension $(2.5 \mu \mathrm{L})$ was incubated in the dark at room temperature for $15 \mathrm{~min}$ with $2 \mathrm{ul} \mathrm{PE}$ CD31 or IgG isotype controls for PE, then they were washed with PBS. For each sample, a total of 20000 events in the EMPs gate were collected and analyzed by using a FACSCalibur flow cytometer. The structure of EMP is observed by TEM.

\section{Assessment of EMP-PDI by flow cytometry}

The HUVEC suspension $(2.5 \mu \mathrm{L})$ was incubated in the dark at room temperature for 15 min with $2 \mu \mathrm{L}$ PE conjugated anti-CD3 1 antibody and $2 \mu \mathrm{L}$ Dylight 488 conjugated PDI antibody, then they were washed with PBS. For each sample, a total of 20000 events in the EMPs gate were collected and analyzed by using a FACSCalibur flow cytometer.

\section{ELISA detection of total amount of EMPs}

ELISA detection of total amount of EMPs was performed as described previously [33]. Briefly, the goat anti Mouse IgG was diluted to $10 \mu \mathrm{g} / \mathrm{mL}$ by package buffer(10mM Tris, $10 \mathrm{mM} \mathrm{NaCl})$, every well was added $50 \mu \mathrm{L}$ goat anti Mouse $\mathrm{IgG}$ for one night at room temperature. After specific capture for microparticles, the plate coated with AD-1 was added to well with $50 \mu \mathrm{L} /$ well at $4{ }^{\circ} \mathrm{C}$ for one night, then after 1 washing with $0.05 \%$ PBST, $50 \mu \mathrm{L}$ of supernatant of HUVECs was added to each well and incubated for $2 \mathrm{~h}$ at $37^{\circ} \mathrm{C}$, after 6 washings, the linear absorbance was recorded at $450 \mathrm{~nm}$ by use of a microplate reader after the addition of substrate solution. Then, let them incubate for $1 \mathrm{~h}$ at $37^{\circ} \mathrm{C}$, the plate was read at $450 \mathrm{~nm}$. Quantification of EMPs is obtained by the difference value of the two value of $450 \mathrm{~nm}$.

\section{Platelet functional studies}

\section{Flow cytometry detection of platelet activation}

We detected the platelet activation after the platelet was incubated with EMPs $(30 \mu \mathrm{g} / \mathrm{ml})$ by flow cytometry. Incubate EMPs with $1 \mu \mathrm{g} / \mathrm{ml}$ RL90, IgG $(1 \mu \mathrm{g} / \mathrm{mL})$, rutin $(10 \mu \mathrm{M})$, rutin $(60 \mu \mathrm{M})$ at $37^{\circ} \mathrm{C}$ for $30 \mathrm{~min}$ respectively, then stimulate platelet with the EMPs. Or incubate EMPs with RGDS $(10 \mu \mathrm{g} / \mathrm{mL})$ with platelet at $37^{\circ} \mathrm{C}$ for $15 \mathrm{~min}$, then incubate the platelet with EMPs. The suspension $(20 \mu \mathrm{L})$ was incubated with $2 \mu \mathrm{L}$ APC-conjugated antiCD62P antibody and $2 \mu \mathrm{L}$ FITC-conjugated PAC-1 antibody in the dark at room temperature for $15 \mathrm{~min}$ then they were washed with PBS. For each sample, a total of 20000 events in the platelet gate were acquired and analyzed by using a FACSCalibur flow cytometry.

\section{Platelet aggregation}

Aggregation was performed as described previously [33]. Briefly, aggregation was assessed by turbidimetry with a dual channel aggregometer, with 2 $\mu \mathrm{mol} / \mathrm{L}$ ADP used as an agonist as. PRP was obtained by centrifuging whole blood at $120 \mathrm{~g}$ for $10 \mathrm{~min}$ at $22^{\circ} \mathrm{C}$, and platelet-poor plasma (PPP) was obtained by centrifuging PRP at $3000 \mathrm{~g}$ for $10 \mathrm{~min}$. The platelet concentration of PRP was adjusted to $2.5 \times 10^{8} / \mathrm{mL}$ by the addition of PPP. An amount of 100\% aggregation was defined as the light transmission of PPP, and $0 \%$ was defined as the light transmission of PRP before the addition of agonists. Then the PRP was stimulated with $2 \mu \mathrm{mol} / \mathrm{L}$ ADP, and the change in light transmission was recorded.

\section{The PDI activity by insulin transhydrogenase assay}

PDI activity was determined using the insulin transhydrogenase assay [34]. Add $250 \mu \mathrm{L}(1 \mathrm{mg} / \mathrm{mL})$ insulin and $50 \mu \mathrm{L}$ human plasma or the cell supernatant incubated with RL90, $\operatorname{IgG}, 10 \mu \mathrm{M}$ and $60 \mu \mathrm{M}$ rutin, then add $10 \mu \mathrm{L}$ DTT $(100 \mathrm{mM})$ to a 96 -wells plate. Mix gently. Detect absorbance at $650 \mathrm{~nm}$ at once and every 10 minutes by a microplate reader for continuous 90 120 minutes. 


\section{The EMP-PDI activity by insulin transhydrogenase assay}

Firstly, the monoclonal antibody AD-1 was bound to a $96-$-well plate, then add $50 \mu \mathrm{L}$ the cell supernatant for 2 hours in $37^{\circ} \mathrm{C}$. After 6 washing, add $100 \mu \mathrm{L}(1 \mathrm{mg} /$ $\mathrm{mL})$ insulin and $3 \mu \mathrm{L}$ DTT $(100 \mathrm{mM})$, to this plate and mix gently. Detect absorbance at $650 \mathrm{~nm}$ at once and every 10 minutes by a microplate reader for continuous $90 \sim 120$ minutes.

\section{Western blot analysis}

HUVEC lysates was subjected to immunoblotting with antibodies against IRS-1 or PI3K or p-Akt or total Akt or $\beta$-actin and platelet lysates was subjected to immunoblotting with antibodies against PDI or $\beta$-actin, followed by anti-IgG horseradish peroxidase-conjugated secondary antibody.

\section{Statistical analysis}

Data are expressed as mean $\pm \mathrm{SD}$ and analyses involved use of GraphPad Prism 5 (La Jolla, CA, USA). Differences among groups were analyzed by one-way

ANOVA followed by Tukey post-hoc test. $P<0.05$ was considered statistically significant. Analyses involved use of SPSS 18.0 (SPSS Inc. Chicago, IL, USA).

\section{ACKNOWLEDGMENTS}

This work was supported by the research grants from the National Basic Research Program of China (973 Program, Grant No. 2013CB530703), the National Natural Science Foundation of China (91439201, $81100605,81270352,81270287,81471036,81570356$, 81300168,81470560 and 81570400), the Natural Science Foundation of Shandong Province (ZR2014HQ037), the Specialized Research Fund for the Doctoral Program of Higher Education (SRFDP 20130131120065), and Key research and development program of Shandong Province (2015GSF118062).

\section{CONFLICTS OF INTEREST}

The authors declare no conflicts of interest.

\section{REFERENCES}

1. McEvoy LK, Laughlin GA, Barrett-Connor E, Bergstrom J, Kritz-Silverstein D, Der-Martirosian C and von Mühlen D. Metabolic syndrome and 16-year cognitive decline in community-dwelling older adults. Ann Epidemiol. 2012; 22:310-317

2. Lockhart PB, Bolger AF, Papapanou PN, Osinbowale O, Trevisan M, Levison ME, Taubert KA, Newburger JW, Gornik HL, Gewitz MH, Wilson WR, Smith SC, Baddour LM and
American Heart Association Rheumatic Fever E, and Kawasaki Disease Committee of the Council on Cardiovascular Disease in the Young, Council on Epidemiology and Prevention, C.uncil on Peripheral Vascular Disease, and Council on Clinical Cardiology. Periodontal disease and atherosclerotic vascular disease: does the evidence support an independent association?: a scientific statement from the American Heart Association. Circulation. 2012; 125:2520-2544.

3. Micucci C, Valli D, Matacchione G and Catalano A. Current perspectives between metabolic syndrome and cancer. Oncotarget. 2016; 7:38959-38972. doi: 10.18632/ oncotarget.8341.

4. Kawada T, Otsuka T, Nakamura T and Kon Y. Relationship between sleep-disordered breathing and metabolic syndrome after adjustment with cardiovascular risk factors. Diabetes Metab Syndr. 2015.

5. Ali Khan H and Mutus B. Protein disulfide isomerase a multifunctional protein with multiple physiological roles. Front Chem. 2014; 2:70.

6. Cho J, Furie BC, Coughlin SR and Furie B. A critical role for extracellular protein disulfide isomerase during thrombus formation in mice. J Clin Invest. 2008; 118:1123-1131.

7. Flaumenhaft R. Protein disulfide isomerase as an antithrombotic target. Trends Cardiovasc Med. 2013; 23:264-268.

8. Edman JC, Ellis L, Blacher RW, Roth RA and Rutter WJ. Sequence of protein disulphide isomerase and implications of its relationship to thioredoxin. Nature. 1985; 317:267-270.

9. $\mathrm{Hu}$ P, Luo B H Integrin $\alpha \mathrm{IIb} \beta 3$ transmembrane domain separation mediatesbi-directional signaling across the plasma membrane [J]. PLoS One, 2015, 10: e0116208.

10. $\mathrm{Hu} \mathrm{P}$, Luo B H. Integrin bi-directional signaling across the plasma membrane [J]. J Cell Physiol, 2013, 228: 306-312.

11. Leader A, Mor-Cohen R, Ram R, Sheptovitsky V, Seligsohn U, Rosenberg $\mathrm{N}$ and Lahav J. The role of protein disulfide isomerase in the post-ligation phase of $\beta 3$ integrin-dependent cell adhesion. Thromb Res. 2015; 136:1259-1265.

12. Shattil SJ, Kim C and Ginsberg MH. The final steps of integrin activation: the end game. Nat Rev Mol Cell Biol. 2010; 11:288-300.

13. Essex DW, Li M, Miller A and Feinman RD. Protein disulfide isomerase and sulfhydryl-dependent pathways in platelet activation. Biochemistry. 2001; 40:6070-6075.

14. Hargett LA, Bauer NN. On the origin of microparticles: From "platelet dust" to mediators of intercellular communication. Pulm Circ. 2013; 3:329-340.

15. Wan SW, Lin CF, Lu YT, Lei HY, Anderson R and Lin YS. Endothelial cell surface expression of protein disulfide isomerase activates $\beta 1$ and $\beta 3$ integrins and facilitates dengue virus infection. J Cell Biochem. 2012; 113:1681-1691. 
16. Lahav J, Jurk K, Hess O, Barnes MJ, Farndale RW, Luboshitz $\mathrm{J}$ and Kehrel BE. Sustained integrin ligation involves extracellular free sulfhydryls and enzymatically catalyzed disulfide exchange. Blood. 2002; 100:2472-2478.

17. Raturi A, Miersch S, Hudson JW and Mutus B. Platelet microparticle-associated protein disulfide isomerase promotes platelet aggregation and inactivates insulin. Biochim Biophys Acta. 2008; 1778:2790-2796.

18. Nieuwdorp M, Stroes ES, Meijers JC and Büller, H. Hypercoagulability in the metabolic syndrome. Curr Opin Pharmacol. 2005; 5:155-159.

19. Santilli F, Vazzana N, Liani R, Guagnano MT and Davì G. Platelet activation in obesity and metabolic syndrome. Obes Rev. 2012; 13:27-42.

20. Kakafika AI, Liberopoulos EN, Karagiannis A, Athyros VG and Mikhailidis DP. Dyslipidaemia, hypercoagulability and the metabolic syndrome. Curr Vasc Pharmacol. 2006; 4:175-183.

21. Calles-Escandon J and Cipolla M. Diabetes and endothelial dysfunction: a clinical perspective. Endocr Rev. 2001; 22:36-52.

22. Walther G, Obert P, Dutheil F, Chapier R, Lesourd B, Naughton G, Courteix D and Vinet A. Metabolic syndrome individuals with and without type 2 diabetes mellitus present generalized vascular dysfunction: cross-sectional study. Arterioscler Thromb Vasc Biol. 2015; 35:1022-1029.

23. Perticone M, Cimellaro A, Maio R, Caroleo B, Sciacqua A, Sesti G and Perticone F. Additive Effect of Non-Alcoholic Fatty Liver Disease on Metabolic Syndrome-Related Endothelial Dysfunction in Hypertensive Patients. Int J Mol Sci. 2016; 17.

24. Koga H, Sugiyama S, Kugiyama K, Watanabe K, Fukushima H, Tanaka T, Sakamoto T, Yoshimura M, Jinnouchi $\mathrm{H}$ and Ogawa H. Elevated levels of VE-cadherinpositive endothelial microparticles in patients with type 2 diabetes mellitus and coronary artery disease. J Am Coll Cardiol. 2005; 45:1622-1630.
25. Werner N, Wassmann S, Ahlers P, Kosiol S and Nickenig G. Circulating CD31+/annexin V+ apoptotic microparticles correlate with coronary endothelial function in patients with coronary artery disease. Arterioscler Thromb Vasc Biol. 2006; 26:112-116.

26. Berezin AE, Kremzer AA, Berezina TA and Martovitskaya YV. The pattern of circulating microparticles in patients with diabetes mellitus with asymptomatic atherosclerosis. Acta Clin Belg. 2016:1-8.

27. Uzan G, Prenant M, Prandini MH, Martin F and Marguerie G. Tissue-specific expression of the platelet GPIIb gene. J Biol Chem. 1991; 266:8932-8939.

28. Cheresh DA. Structural and biologic properties of integrinmediated cell adhesion. Clin Lab Med. 1992; 12:217-236.

29. Manickam N, Sun X, Li M, Gazitt Y and Essex DW. Protein disulphide isomerase in platelet function. Br J Haematol. 2008; 140:223-229.

30. Cho J, Kennedy DR, Lin L, Huang M, Merrill-Skoloff G, Furie BC and Furie B. Protein disulfide isomerase capture during thrombus formation in vivo depends on the presence of ?23 integrins. Blood. 2012; 120:647-655.

31. Jasuja R, Furie B and Furie BC. Endothelium-derived but not platelet-derived protein disulfide isomerase is required for thrombus formation in vivo. Blood. 2010; 116:4665-4674.

32. Kim K, Hahm E, Li J, Holbrook LM, Sasikumar P, Stanley RG, Ushio-Fukai M, Gibbins JM and Cho J. Platelet protein disulfide isomerase is required for thrombus formation but not for hemostasis in mice. Blood. 2013; 122:1052-1061.

33. Wang H, Wang ZH, Kong J, Yang MY, Jiang GH, Wang XP, Zhong M, Zhang Y, Deng JT and Zhang W. Oxidized low-density lipoprotein-dependent platelet-derived microvesicles trigger procoagulant effects and amplify oxidative stress. Mol Med. 2012; 18:159-166.

34. Holmgren A. Thioredoxin catalyzes the reduction of insulin disulfides by dithiothreitol and dihydrolipoamide. J Biol Chem. 1979; 254:9627-9632. 